\title{
Un nuevo método sencillo y exacto para medir el tamaño de los pólipos durante la colonoscopia
}

\section{A simple new method to accurately measure the size of polyps during colonoscopy}

William Otero Regino, MD, ${ }^{1}$ Liliana Oino Embus, MD, ${ }^{2}$ Lina Parra Otero, MD³

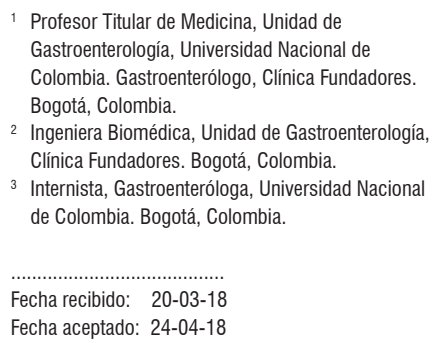

\begin{abstract}
Resumen
Introducción: tradicionalmente, los pólipos colónicos se miden empíricamente por estimación visual y con las pinzas de biopsia, aunque dichos métodos son inexactos. Diferentes métodos han sido investigados, pero no tienen la exactitud esperada. Por lo anterior, se realizó este trabajo para construir un algoritmo que permitiera medir los pólipos a partir de fotografías tomadas con el colonoscopio. Materiales y métodos: el trabajo se realizó en tres fases. En la primera, se construyó un algoritmo en el programa MATLAB. Se capturaron fotos en formato JPG con el colonoscopio. En la segunda fase, con el algoritmo se midieron imágenes de objetos con tamaños conocidos para verificar la exactitud del algoritmo. Después de verificar la exactitud, fue sometida al algoritmo la fotografía de los pólipos del colon. En la tercera fase, se utilizaron imágenes de pólipos previamente enviadas a tres expertos. Todas las fotografías fueron tomadas con el colonoscopio Olympus Exera II. Resultados: en los objetos menores de $5 \mathrm{~mm}$, el algoritmo sobreestimó el tamaño entre 0,11 y 0,08 mm; en los mayores de $5 \mathrm{~mm}$, sobreestimó el tamaño entre $0,25 \mathrm{~mm}$ y $1,76 \mathrm{~mm}$ en los de $22 \mathrm{~mm}$. Los expertos sobrestimaron los tamaños de manera importante. En los pólipos de 7, 8 y $9 \mathrm{~mm}$, los expertos dijeron que medían 12, 15 y $18 \mathrm{~mm}$, respectivamente. Conclusión: el algoritmo desarrollado tiene adecuada exactitud para medir pólipos colónicos. Por su fácil consecución y utilización, podría ser una herramienta para solucionar la dificultad de medir pólipos durante una colonoscopia.
\end{abstract}

\section{Palabras clave}

Algoritmo, MATLAB, pólipos de colon, pinza de biopsia, píxeles.

\begin{abstract}
Introduction: Traditionally, colon polyps' measurements have been empirically estimated visually and with biopsy forceps, but neither method is inaccurate. Other methods have been studied but have not had the accuracy expected. The research reported here was undertaken to address this issue by building an algorithm for measure polyps from photographs taken through a colonoscope. Materials and methods: The study was done in three phases. First, an algorithm was built in MATLAB, and photos taken with a colonoscope were stored in the JPG format. In the second phase, images of objects with known sizes were checked against the algorithm to verify its accuracy. After verification of the algorithm's accuracy, photographs of colon polyps were measured using the algorithm. In phase 3 , images of polyps previously sent to three experts were measured with the algorithm. All photographs were taken with an Olympus Exera II Colonoscope. Results: For objects smaller than $5 \mathrm{~mm}$, the algorithm overestimated sizes by 0.11 to $0.08 \mathrm{~mm}$. For those greater than $5 \mathrm{~mm}$, it overestimated sizes by $0.25 \mathrm{~mm}$ to $1.76 \mathrm{~mm}$ in those of $22 \mathrm{~mm}$. The experts seriously overestimated sizes. They estimated that $7 \mathrm{~mm}$ polyps measured $12 \mathrm{~mm}, 8 \mathrm{~mm}$ polyps measured $15 \mathrm{~mm}$, and $9 \mathrm{~mm}$ polyps measured $18 \mathrm{~mm}$. Conclusion: The algorithm developed is sufficiently accurate for measuring colon polyps and is easy to obtain and relatively easy to use. It could become a tool for overcoming the difficulty of measuring polyps during a colonoscopy.
\end{abstract}

\section{Keywords}

Algorithm, MATLAB, colon polyps, biopsy forceps, pixels. 


\section{INTRODUCCIÓN}

A nivel mundial, el cáncer de colon y recto (CCR) es el tercer cáncer más común en varones y el segundo más común en mujeres (1). En 2012, hubo 1,4 millones de casos nuevos y 694000 muertes (2). Representa la tercera causa más común de muerte por cáncer en el mundo (2). En Colombia, el CCR también es la cuarta causa más frecuente de muerte por cáncer, tanto en varones como en mujeres (3). En las últimas décadas, el CCR ha aumentado en los países desarrollados, en contraste con los países subdesarrollados, en los que ha permanecido estable o incluso ha disminuido (1, 3, 4). La incidencia es más alta en Australia, Nueva Zelanda y Europa Occidental y más baja en África Central y en el Centro y Sur de Asia (1). La mayoría de los CCR se originan en los pólipos adenomatosos del colon siguiendo la secuencia de pólipo-adenocarcinoma (5). Dependiendo del tamaño, el genoma de esas lesiones puede presentar mutaciones que inician en el gen APC (Adenomatous Polyposis Coli) y después en los genes KRAS y p53 (6). El sinergismo de esa mutación determina que los pólipos adenomatosos pequeños y los de mayor tamaño se transformen en CCR (6). Este conocimiento es la base para la colonoscopia de "screening" o tamización en personas de 50 años o más, para disminuir la mortalidad por CCR con la detección y resección de los pólipos encontrados $(7,8$, 9). Dependiendo del tamaño de los pólipos, se determinan los intervalos de vigilancia de los pacientes $(10,11)$.

Sin embargo, en la actualidad, la estimación del tamaño de los pólipos sigue siendo inexacta. La mayoría de los colonoscopistas "miden" el tamaño de los pólipos por "estimación visual", la cual es inexacta, incluso entre expertos, y se puede sobreestimar o subestimar el verdadero tamaño $(12,13)$. Además, la medición que se hace en patología con el espécimen resecado es inferior a la que se hace in vivo $(14,15$, $16,17)$. Cuando el tamaño es subestimado, erróneamente se pueden recomendar intervalos de vigilancia muy largos, que aumentan el riesgo de aparición de CCR de intervalo (18). Múltiples investigadores han estudiado la forma de medir los pólipos, incluso con tomografía axial computarizada (TAC) $(13,14,15,16,17,18)$. Entre los diferentes métodos, el más sencillo y más frecuentemente usado es el de abrir una pinza de biopsias cerca al pólipo, y como el tamaño de la pinza abierta es conocido $(5-6 \mathrm{~mm})$, se calcula el tamaño del pólipo superponiendo la pinza "cerca" del pólipo. Sin embargo, el margen de error de este "método" es cercano al $50 \%$, hacia arriba o hacia abajo, incluso entre expertos (13). En un estudio reciente que usó este método, la determinación del tamaño del pólipo fue correcta en el $37 \%$ de los pólipos; la medición fue más grande en el $34 \%$ y más pequeña en el 29 \% (13). Otros métodos utilizados incluyen una "capucha” calibrada, que se coloca en la punta del colonoscopio
(19). Con este método se demostró que la estimación visual es inexacta, en contraste con el aditamento utilizado para la investigación, que fue más exacto, pero difícil de implementar. Recientemente se ha propuesto un nuevo método complejo y sofisticado, con una sonda óptica y un computador personal, para generar una escala de grises que permita determinar el tamaño de los pólipos a partir de la imagen endoscópica y de acuerdo con la distancia entre los objetos (20). En esta investigación, la amplitud de la escala de grises fue ajustada constantemente de acuerdo con la distancia entre la punta del colonoscopio y la lesión, ya que el tamaño de la lesión varía de acuerdo con la distancia. La distancia entre los objetos fue calculada según la cantidad de luz láser reflejada de la lesión a través de una sonda óptica insertada en el canal del endoscopio. Los resultados de este método permitieron concluir que, con el endoscopio convencional, se podría utilizar este sistema para lograr una determinación más exacta del tamaño. Sin embargo, el costo y la dificultad para su implementación no lo hacen viable. La medición correcta del tamaño del pólipo tiene dos implicaciones: 1 . Elegir más eficazmente el tamaño del asa y la técnica para la polipectomía; y 2. Recomendar correctamente los intervalos de vigilancia, ya que, si se encuentran uno o más adenomas con tamaños menores de $10 \mathrm{~mm}$, la vigilancia es similar a la tamización, cada diez años; en cambio, si son de tres a cuatro pequeños o al menos uno de $10-19 \mathrm{~mm}$, será en tres años; y si son más de cinco pólipos pequeños, o uno de $20 \mathrm{~mm}$ o más, la próxima colonoscopia será en un año (21).

Teniendo en cuenta la necesidad de medir correctamente los pólipos y la ausencia de un método ampliamente disponible, decidimos realizar el presente trabajo con los siguientes objetivos.

\section{OBJETIVO GENERAL}

Diseñar un método para medir los pólipos del colon durante una colonoscopia de rutina, mediante un algoritmo con el software matemático MATLAB.

\section{Objetivos específicos}

1. Utilizar el algoritmo desarrollado para medir objetos con tamaños conocidos y determinar la exactitud de estos.

2. Someter las imágenes de los pólipos del colon al algoritmo y estimar el tamaño.

3. Comparar las medidas de pólipos colónicos estimadas visualmente por tres expertos y comprarlas con el algoritmo de MATLAB.

4. Instalar en los computadores de la Unidad de Gastroenterología la parte específica de MATLAB con el algoritmo, para utilizar este método en la práctica rutinaria. 


\section{MATERIALES Y MÉTODOS}

El presente trabajo fue realizado en la Unidad de Gastroenterología de la Clínica Fundadores de Bogotá, Colombia, durante el período comprendido entre agosto de 2014 y agosto de 2016, descrito a continuación. En la primera fase se desarrolló un algoritmo en el software matemático MATLAB y se capturaron fotos en formato JPG con un colonoscopio Olympus Exera II. En la segunda fase, se sometieron al algoritmo imágenes de objetos con tamaños conocidos y se verificó su exactitud. Una vez comprobado el buen funcionamiento del algoritmo, se utilizaron fotos de pólipos para determinar sus tamaños con el algoritmo desarrollado. La tercera fase consistió en someter al algoritmo las imágenes de pólipos que previamente habían sido enviados a tres expertos en gastroenterología, para que estimaran visualmente el tamaño de estos. El propósito de esta última fase era determinar qué tan exacta es la estimación visual de los expertos comparándola con las medidas del método desarrollado. Todas las fotografías sometidas al algoritmo de MATLAB fueron tomadas con un colonoscopio Olympus Exera II en formato JPG, con protocolo estándar. Ese protocolo consistía en capturar las imágenes a la distancia producida cuando por la punta del colonoscopio sobresalía una pinza de biopsia marca Olympus y se le visualizaban tres anillos. Se eligió dicha distancia porque encontramos que permite una adecuada visualización de las lesiones. Las pinzas de biopsias utilizadas fueron las pinzas Olympus reusables, que están hechas en acero y cuya cubierta es anillada. Para los propósitos de esta investigación, era imprescindible un adecuado posicionamiento cuando se visualizaban tres anillos de esta en el monitor. Con esta técnica, la distancia estandarizada que elegimos tenía $13 \mathrm{~mm}$. Igualmente se puede utilizar cualquier otro dispositivo para estandarizar la distancia, la cual se suministrará al algoritmo matemático y se mantiene la exactitud de la medida. Para desarrollar el algoritmo, se contrataron los servicios profesionales de un ingeniero de sistemas experto en MATLAB, a quien le informamos sobre nuestros objetivos y el interés en utilizar el software matemático mencionado por contener "las herramientas" para este tipo de diseños. Uno de nosotros es ingeniero biomédico (LOE).

La preparación de la colonoscopia se realizó en dosis divididas (Split), en la forma usual (9). Brevemente, el día anterior al examen, el paciente tuvo dieta normal hasta el almuerzo y, de ahí en adelante, dieta líquida. A las 4.00 p. m. de ese día, se inició la preparación para la limpieza del colon utilizando dos sobres de polietilenglicol con electrólitos (Nulytely ${ }^{\oplus}$ ). Cada sobre fue disuelto en 1 litro de agua. Durante dos horas, el paciente ingería un vaso de la solución $(250 \mathrm{~mL})$ cada 15 minutos, de tal manera que debía consumir 1 litro por hora durante dos horas. El día del exa- men, a las 7.00 a. m., se reanudó la ingesta de los otros dos sobres de la preparación, que fueron disueltos de manera similar al día anterior; es decir, cada sobre en 1 litro de agua. La preparación se terminaba a las 9.00 a. m. Dos a tres horas después, se llevó a cabo la colonoscopia sin sedación, en decúbito lateral izquierdo o en decúbito supino. Los procedimientos fueron ejecutados por un colonoscopista experto, profesor universitario (WOR). Las colonoscopias se realizaron con un equipo Olympus 180 Exera II.

\section{Consideraciones éticas}

Según la Resolución 008430 de 1993, este trabajo es una investigación de bajo riesgo, ya que se utilizan datos externos a los pacientes y algunos son tomados de un examen de rutina independiente de la investigación. Dada la dificultad que puede existir con la terminología relacionada con los programas de computación, consideramos de utilidad las siguientes definiciones básicas.

\section{Imagen digital}

Una imagen digital es una fotografía, un dibujo, un trabajo artístico o cualquier otra "imagen" que es convertida en un fichero de ordenador. Una imagen digital consiste en una colección ordenada de valores. Estos valores representan una colección de filas de valores dispuestas ordenadamente (22).

\section{Formato de imagen}

Las imágenes digitales se pueden guardar en distintos formatos. Cada uno se corresponde con una extensión específica del archivo que lo contiene. Los más utilizados en la actualidad son: BMP, GIF, JPG, TIF y PNG (23).

\section{Píxel (24)}

Es la unidad mínima de visualización de una imagen digital. Las cámaras digitales y los escáneres capturan las imágenes en forma de cuadrícula de píxeles. Los píxeles son aquellos puntos que forman las imágenes digitales. Cada uno de estos puntos se denomina píxel, y al observarlos todos juntos se forma una imagen. La cantidad de puntos o píxeles con que cuente una imagen indicará la calidad de su resolución. En términos sencillos, son los "puntitos" con los que están hechas las imágenes en el mundo de la computación. Para almacenar la información de una imagen, cada píxel se codifica mediante un conjunto de bits de una longitud determinada (llamada profundidad de color). Por ejemplo, un solo píxel puede codificarse con una profundidad de color de 8 bits ( 1 byte), y esto permite que pueda tomar hasta 256 variantes de color ( 2 elevado a la 8 ). En las imágenes fotográficas, se suelen usar 3 bytes ( 24 bits) para definir cada color de cada píxel. Con esto pueden representarse 16'777 216 de colores. Este tipo de imágenes se denominan true color. 
Los píxeles también se utilizan como unidad para medir la resolución de una pantalla, una imagen y de algunos dispositivos como, por ejemplo, las cámaras digitales (que utilizan los megapíxeles). En cuanto a las imágenes, estas pueden medirse a través del ancho y del largo en píxeles; por ejemplo, una imagen de $800 \times 600$, lo que significa que está conformada por 480000 píxeles (25).

\section{Algoritmo (26)}

En matemáticas, lógica, ciencias de la computación y disciplinas relacionadas, un algoritmo (del griego y latín dixit algorithmus, y este, a su vez, del matemático persa Al-Juarismi) es un conjunto prescrito de instrucciones o reglas bien definidas, ordenadas y finitas que permite realizar una actividad mediante pasos sucesivos que no generen dudas a quien deba realizar dicha actividad. Dados un estado inicial y una entrada, siguiendo los pasos sucesivos, se llega a un estado final y se obtiene una solución. Los algoritmos son el objeto de estudio de la algoritmia (26).

\section{MATLAB}

Es el nombre abreviado de MATrix LABoratory, desarrollado por MathWorks (27). MATLAB es un software matemático muy versátil en sus aplicaciones matemáticas. Es una de las muchas herramientas sofisticadas de computación disponibles en el comercio e incluso puede descargarse por internet. Permite resolver problemas de matemáticas, tales como Maple, Mathematica y MathCad (28). Ofrece un entorno de desarrollo integrado con un lenguaje de programación propio. Con MATLAB es posible realizar cálculos numéricos con vectores y matrices. Puede también trabajar con números escalares, tanto reales como complejos, con cadenas de caracteres y con otras estructuras de información más complejas. Una de las capacidades más atractivas que tiene es la de realizar una amplia variedad de gráficos en dos y tres dimensiones. MATLAB fue originalmente una herramienta para la realización de álgebra matricial. Con el tiempo, se ha convertido en un entorno de programación con varias partes. Entre ellas se encuentran los gráficos tridimensionales, las interfaces gráficas de usuario, la interacción con otras descargas y las cajas de herramientas. Las cajas de herramientas son los paquetes de programas que proporcionan funcionalidad adicional a MATLAB (28).

\section{MATLAB en ingeniería biomédica $(29,30)$}

Por lo general, las imágenes médicas se guardan como archivos dicom (el estándar Digital Imaging and Communications in Medicine: imágenes digitales y comunicaciones en medicina). Los archivos dicom utilizan la extensión de archivo .$d c m$. La compañía MathWorks ofrece una caja de herramientas adicional, llamada caja de herramientas para imágenes, que puede leer esos archivos. Esto permite que sus datos estén disponibles para procesamiento en MATLAB. La caja de herramientas para imágenes también incluye un amplio rango de funciones de las que muchas son especialmente apropiadas para las imágenes médicas. Un conjunto limitado de datos MRI ya convertidos a un formato compatible con MATLAB se incluye con el programa MATLAB estándar. Este conjunto de datos le permite probar algunas de las funciones de generación de imágenes disponibles tanto con la instalación estándar de MATLAB como con la caja de herramientas para imágenes expandida. MATLAB dispone de múltiples herramientas, con funciones específicas que incluyen imdistline, subplot, uigetfile, imread, entre otras.

\section{FASES DE LA INVESTIGACIÓN}

\section{Fase 1}

Esta fase se inició tomando fotografías con el colonoscopio, en formato JPG, de los objetos de referencia. Estos objetos tenían tamaños conocidos y suministrados por el fabricante. Los elementos utilizados, fueron anillos de caucho, que fueron elaborados específicamente para la presente investigación, en la fábrica Discorreas, Mangueras y Empaques S.A., de Bogotá. Cada anillo tenía su respectivo código y certificación sobre el diámetro externo y diámetro interno. Los tamaños de los objetos de referencia se muestran en la Tabla 1.

Tabla 1. Anillos de caucho con diámetros conocidos (patrones de referencia)

\begin{tabular}{ccc}
\hline $\begin{array}{c}\text { Referencia } \\
\text { Objeto }\end{array}$ & $\begin{array}{c}\text { Diámetro interno } \\
(\mathrm{mm})\end{array}$ & $\begin{array}{c}\text { Diámetro externo } \\
(\mathrm{mm})\end{array}$ \\
\hline $2-001$ & 0,74 & 2,78 \\
$2-002$ & 1,07 & 3,61 \\
$2-003$ & 1,42 & 4,46 \\
$2-004$ & 1,78 & 5,34 \\
$2-005$ & 2,57 & 6,13 \\
$2-006$ & 2,90 & 6,46 \\
$2-012$ & 9,25 & 12,81 \\
\hline
\end{tabular}

La distancia a la cual se tomaron las fotos de estos elementos es la misma que se utilizó para tomar las fotos de los pólipos, como fue descrito previamente. Si bien esta distancia no es utilizada para hacer los cálculos, ni influye en el desarrollo del algoritmo, se utilizó para estandarizar la captura de la imagen y la fotografía, con el fin de que cada foto capturada tuviera la misma distancia para que fueran comparables entre sí y se evitaran los sesgos (Figura 1).

Para estimar los tamaños, de los diferentes objetos, se diseñó un algoritmo sencillo (para los ingenieros expertos) utilizando diferentes funciones contenidas en la caja de herramientas de MATLAB. 


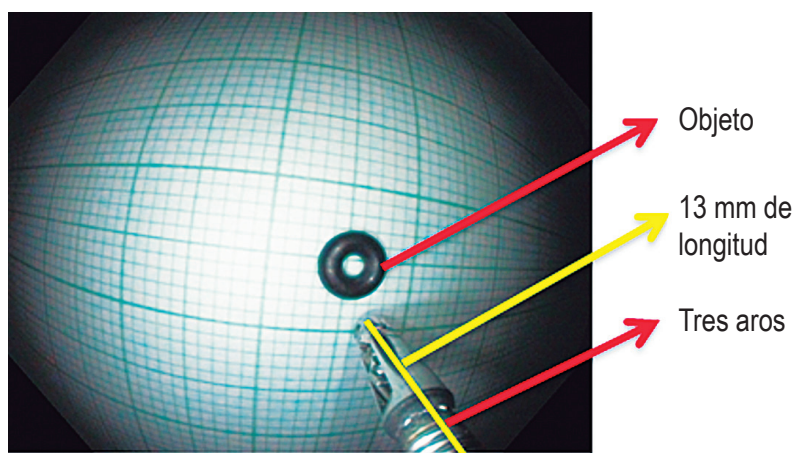

Figura 1. Patrón de referencia con imagen capturada por el colonoscopio.

En primera instancia, se verificó que el algoritmo fuera eficiente utilizando diferentes fotografías en formato JPG. Se consideró que el programa corría adecuadamente cuando permitía cargar la imagen y manipular imdistline. Sucesivamente, el programa pide ingresar un valor en píxeles y finalmente proporciona o "arroja" un resultado. En la secuencia del funcionamiento básicamente se utilizaron las siguientes funciones: "uigetfile", "imread", "strcat", "imshow", "subplot", "imdistline", "Inputdlg", "str2double".

Con base en las funciones previas, se crearon cuatro variables. Dos de ellas (A y B) tenían valores hipotéticos que se suministraron para que el algoritmo calculara el valor de la cuarta variable (D), después de suministrarle el valor de la tercera $(C)$ mediante una regla de tres simple. La variable A guarda un dato fijo en píxeles, la variable $B$ almacena un dato fijo en milímetros, la variable $\mathrm{C}$ guarda un valor que el usuario ingresa y la variable $\mathrm{D}$ realiza una regla de tres con los datos almacenados en las variables A, B y C.

La función "msgbox" finalmente muestra en milímetros el resultado que fue calculado en el proceso anterior. Una vez se verificó el correcto funcionamiento del algoritmo, se determinó a cuántos píxeles equivale una determinada área; en este caso, $5 \mathrm{~mm}$. Para encontrar este valor, se le tomó una fotografía en formato JPG a una hoja milimetrada de manera similar a la tomada a los anillos de caucho. En dicha hoja, se incluyó un anillo de caucho con tamaño conocido. $\mathrm{Al}$ algoritmo establecido se le sometió la fotografía mencionada. Se escogieron los cuatro cuadrantes cercanos a la posición de la pinza de biopsias debido a que esta es la posición donde se visualiza el pólipo, y usando la función "imdistline" se trazó una línea horizontal sobre cada uno de los cuadrantes de las líneas de la cuadrícula con tamaño conocido $(5 \mathrm{~mm})$ y se obtuvo el valor del píxel. Esta foto tomada por nosotros se muestra en la Figura 2. La cuadrícula derecha, que está compuesta por un cuadrante supe-
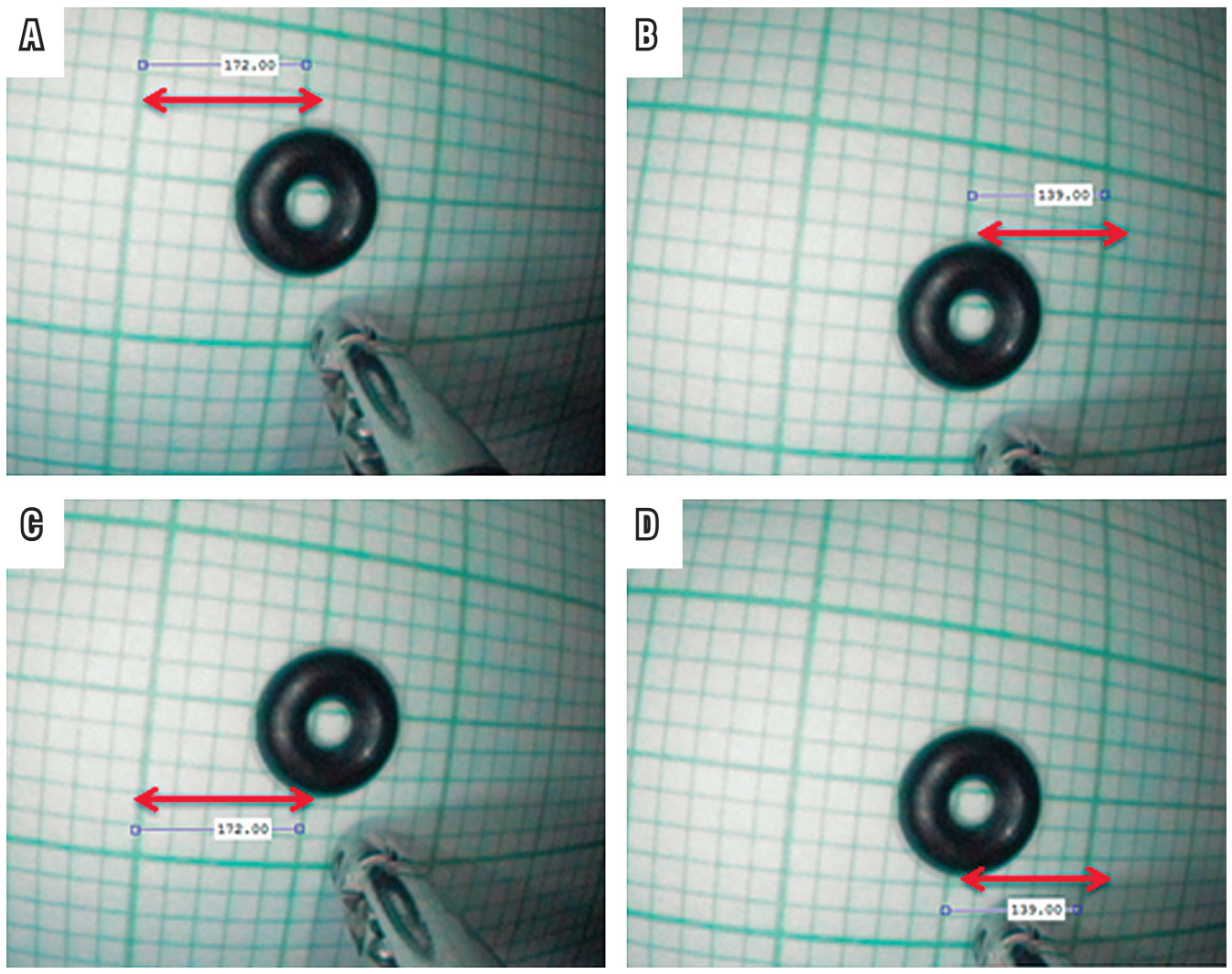

A. Valor en píxeles del cuadrante superior izquierdo (172,00 pixeles).

B. Valor en píxeles del cuadrante superior derecho $(139,00$ pixeles).

C. Valor en píxeles del cuadrante inferior izquierdo (172,00 pixeles).

D. Valor en píxeles del cuadrante inferior derecho (139,00 pixeles).

Figura 2. Valor en píxeles de cada uno de los cuadrantes. 
rior e inferior, comparte el mismo valor de 172,00 píxeles, y la cuadrícula izquierda, que también está compuesta por un cuadrante superior e inferior, tiene el mismo tamaño en píxeles de 139,00. El valor de las cuadrículas en pixeles es diferente debido a que la imagen es convexa.

Los valores obtenidos en píxeles de cada uno de los cuadrantes de $5 \mathrm{~mm}$ y el promedio de estos quedaron guardados en el algoritmo como el patrón de referencia. Estos valores se muestran en la Tabla 2.

Tabla 2. Valores en píxeles de los cuadrantes de $5 \mathrm{~mm}$

\begin{tabular}{lcc}
\hline $\begin{array}{c}\text { Lado de la cuadrícula } \\
\text { medida }\end{array}$ & $\begin{array}{c}\text { Valor en } \\
\text { píxeles }\end{array}$ & $\begin{array}{c}\text { Tamaño de la cuadrícula } \\
\text { en milímetros (mm) }\end{array}$ \\
\hline Cuadrante superior derecho & 172,00 & $5 \mathrm{~mm}$ \\
Cuadrante superior izquierdo & 139,00 & $5 \mathrm{~mm}$ \\
Cuadrante inferior derecho & 172,00 & $5 \mathrm{~mm}$ \\
Cuadrante inferior izquierdo & 139,00 & $5 \mathrm{~mm}$ \\
Promedio en píxeles & 155,5 & $5 \mathrm{~mm}$ \\
\hline
\end{tabular}

En esta primera fase, también se verificó la resolución de las fotografías con respecto a las dimensiones en píxeles y a la profundidad en bits, como se muestra en dos diferentes fotografías (A y B) de la Figura 2, en las que se verificó que todas las fotos tomadas de la manera estandarizada contenían la información en píxeles y la profundidad, independientemente del color de la imagen y del color del fondo. Esto contrasta con el método de segmentación de imágenes, en el cual, el objeto de estudio queda inmerso en el entorno y no se puede resaltar claramente con más o menos píxeles. Con el método elegido en esta investigación, la información depende de la distancia en que se captura la imagen durante la fotografía, ya que el dispositivo acoplado a la carga (CCD) de la cámara del colonoscopio tiene la propiedad de que todas las fotos tomadas tengan la misma profundidad en bits, que en este caso es de 24 bits, como se muestra en la Figura 3.

No se llevaron a cabo procesamientos de imágenes o segmentación de estas, aunque el MATLAB lo dispone, debido a que los píxeles contenidos dentro del objeto, que, en cuyo caso, son los pólipos, son similares o iguales a los que están en el fondo del objeto (mucosa del colon).

\section{Fase 2}

El patrón de referencia final del algoritmo, que fue de 155,5 píxeles contenidos en una cuadrícula de $5 \mathrm{~mm}$ (véase Tabla 2), permitirá calcular en adelante las imágenes que en formato JPG se le ingresen. En esta fase, se sometieron las fotografías de los anillos de caucho con tamaños conocidos y adicionalmente otros objetos como monedas de diferente nominación y un lunar de la piel de una persona voluntaria. Después de cargar la imagen en el algoritmo, se utilizó la función "imdistline" y se midió el diámetro
A

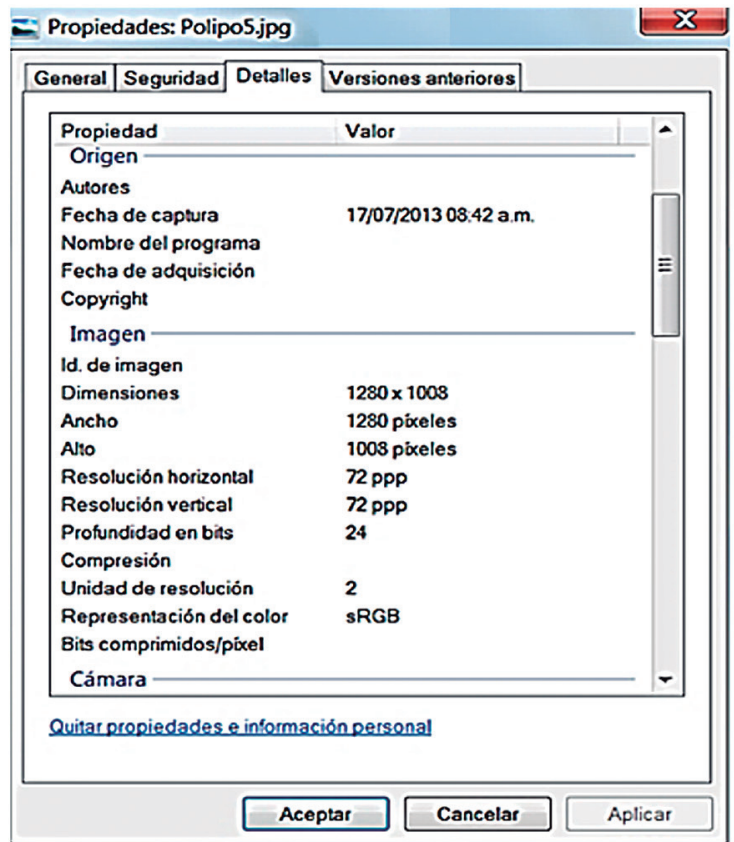

B

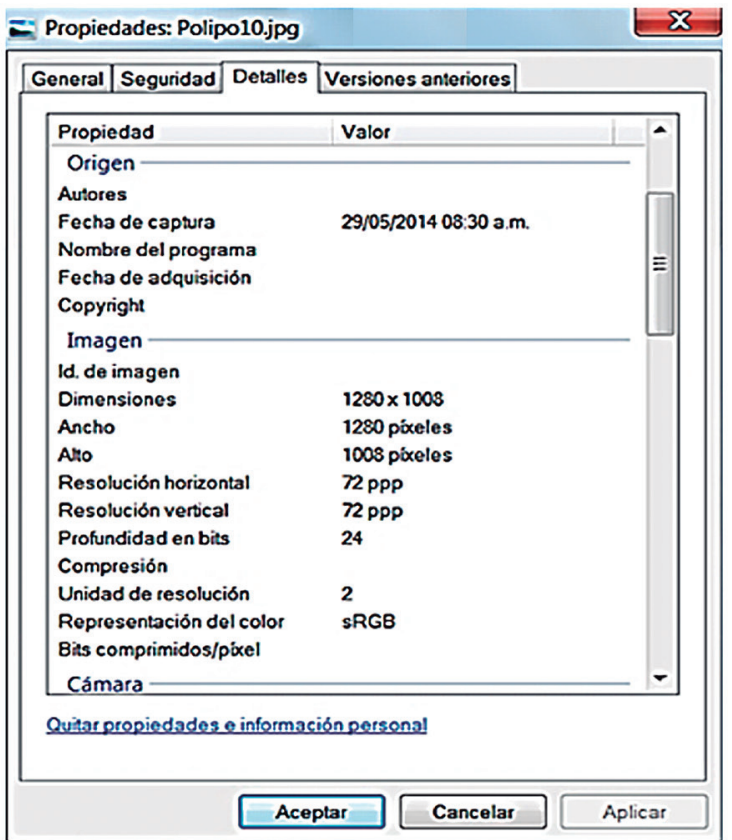

Figura 3. Dimensiones en píxeles y profundidad en bits de dos fotografías diferentes. 
del objeto trazando una línea de un punto a otro punto sobre el objeto en estudio (Figuras 4, 5, 6 y 7). La función "inputdlg", por medio de una ventana, permite el ingreso del valor en píxeles previamente visto (Figuras 4C, 5C y 6C). Después, arroja el valor en milímetros (Figuras 4D, 5D y 6D). Igualmente se sometieron al algoritmo otros elementos con diámetros conocidos, tales como monedas de diferentes nominaciones, pinza de biopsia abierta, un lunar de la piel y pólipos del colon, cuando se estableció la exactitud del método para medir los diferentes objetos. En las Figuras 4, 5 y 6 se muestran los objetos.

En esta fase también se sometieron varios pólipos y en la Figura 7 se muestra uno de estos, que corresponde a un pólipo pediculado.

\section{Fase 3}

Después de encontrar que el algoritmo construido en MATLAB tenía una excelente concordancia, fue considerado como la prueba de referencia para la tercera fase. En esta fase se enviaron fotografías en formato JPG, tomadas con la misma metodología descrita, a tres gastroenterólogos expertos para que estimaran visualmente el tamaño de estos. Los tamaños estimados visualmente por ellos fueron confrontaron con los arrojados por el algoritmo.

\section{RESULTADOS}

Los valores en píxeles y en milímetros, calculados por el algoritmo de MATLAB, se muestran en las Figuras 5, 6 y 8. Sus valores consolidados se muestran en la Tabla 3. En esa misma tabla se comparan los valores de cada elemento que arrojó el algoritmo de MATLAB con los valores reales de cada objeto, incluida la lesión de la piel, y se muestran las variaciones de estas dos medidas. Los signos (+) y (-) hacen referencia al tamaño suministrado por MATLAB, cuando es más o es menos que el valor real.

En esta tabla se observa que el algoritmo sobreestima el tamaño real entre 0,11 y $0,08 \mathrm{~mm}$, cuando el objeto mide menos de $5 \mathrm{~mm}$, y da un tamaño ligeramente superior (la décima parte de $1 \mathrm{~mm}$ ). En cambio, subestima (da un valor inferior) en $0,25 \mathrm{~mm}$ los objetos mayores de $5 \mathrm{~mm}$. Cuando el objeto mide $22 \mathrm{~mm}$, el error es de $1,76 \mathrm{~mm}$. Con estas mínimas variaciones, la concordancia kappa es casi perfecta.

Después de haber verificado la exactitud del algoritmo cuando se midieron los objetos con valores reales, se calculó

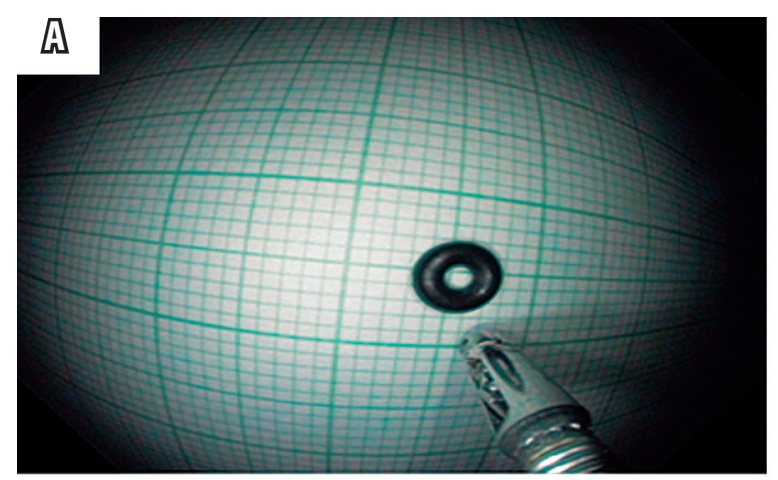

C
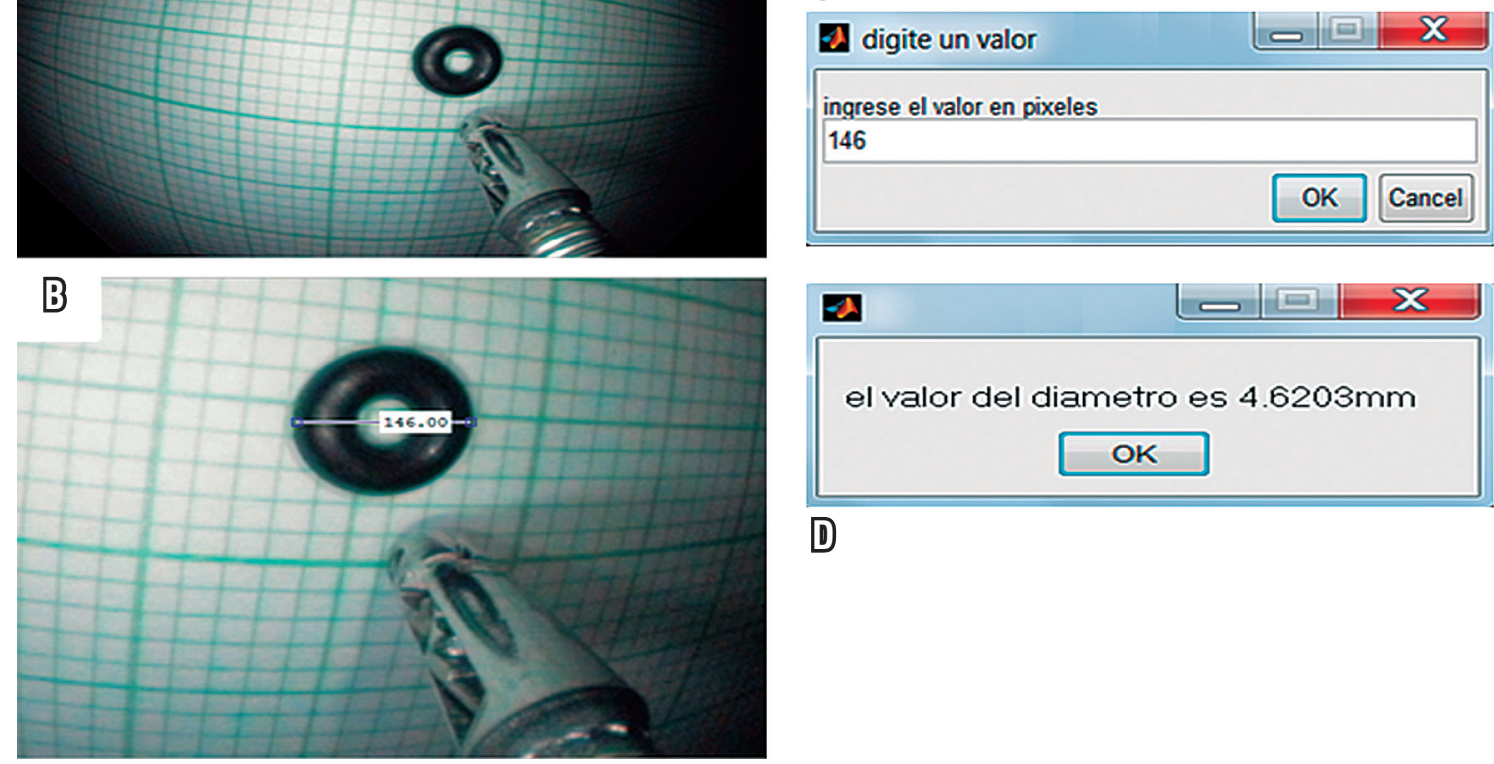

D

Figura 4. Cálculo del tamaño de un objeto conocido en milímetros. A. Foto de un objeto conocido (anillo de caucho), ya ingresada al algoritmo. B. Haciendo uso de la función "imdistline”, se utiliza el proceso ya conocido, se le traza la línea y se obtiene el valor en píxeles. C. Se ingresa el valor en píxeles y dando clic en "OK” arroja el resultado en milímetros. 


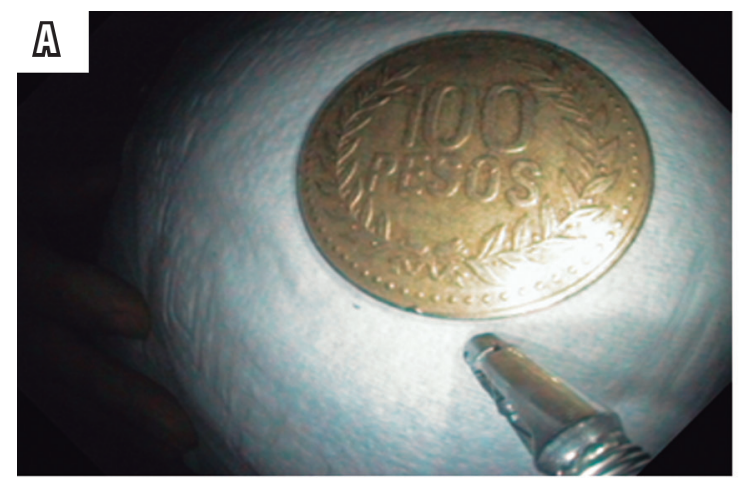

\section{C}

\begin{tabular}{|l|l|l|l|}
\hline digite un valor & & & \\
\hline ingrese el valor en pixeles & \\
\hline 629.76 & & \\
\hline & OK & Cancel \\
\hline
\end{tabular}
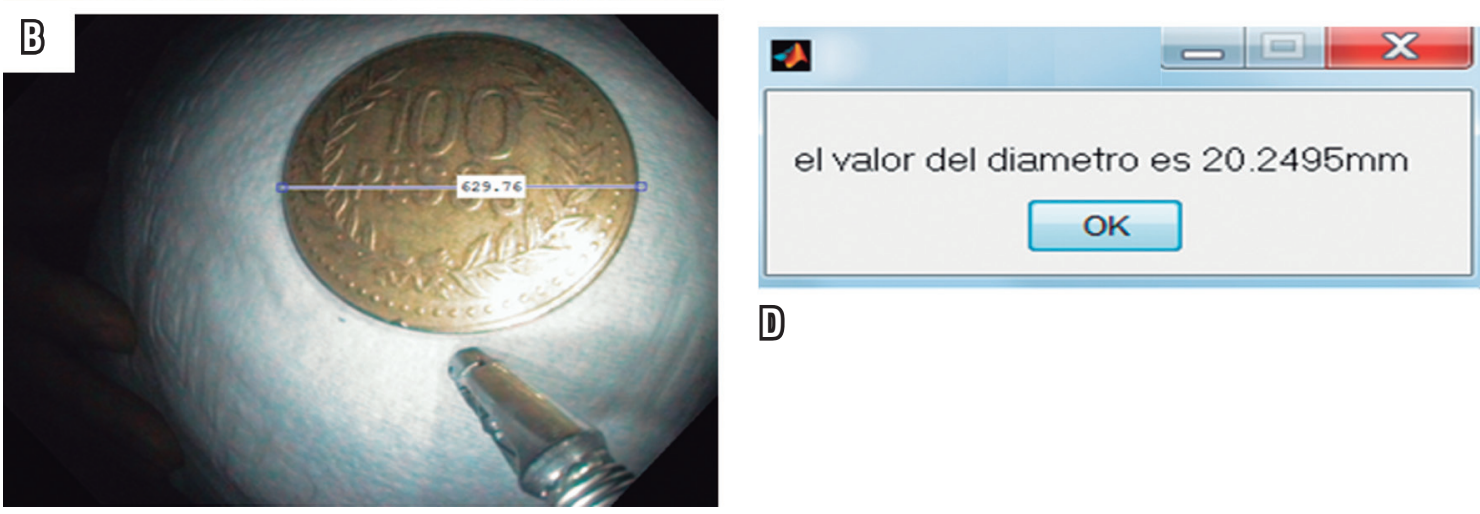

D

Figura 5. Cálculo del tamaño en milímetros de un objeto conocido. A. Objeto con tamaño conocido (moneda de 100 pesos). B. Medida en píxeles con la herramienta "imdistline". C. Ventana para ingresar el valor en píxeles. D. Ventana de resultado del diámetro del objeto en milímetros.

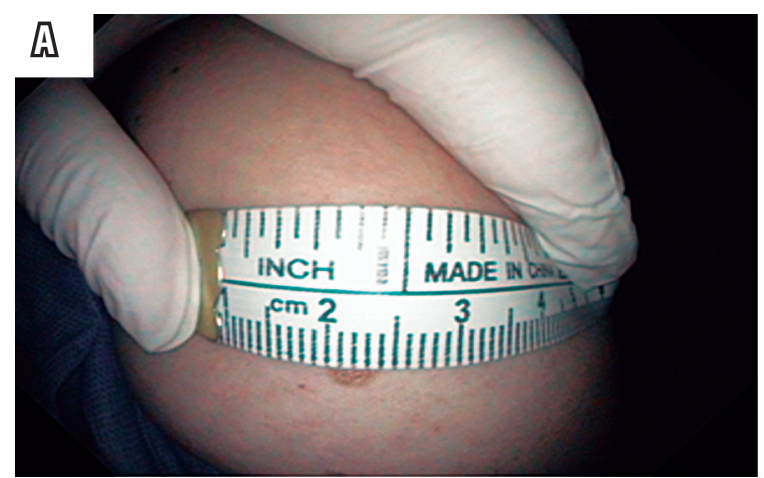

ㄴ

B

\begin{tabular}{|l|l|l|l|}
\hline digite un valor & & \\
\hline ingrese el valor en pixeles & \\
\hline 128.02 & & \\
\hline & OK & Cancel \\
\hline
\end{tabular}

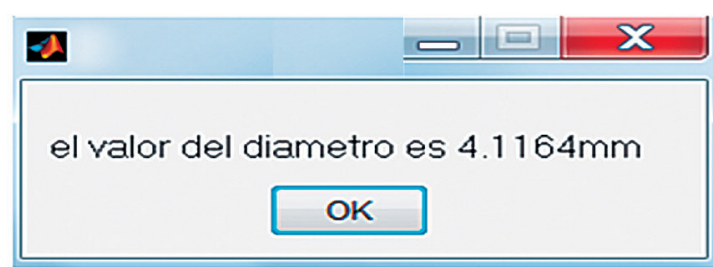

D

Figura 6. A. Lesión de la piel con tamaño conocido (lunar). B. Medida en píxeles con la herramienta "imdistline". C. Ventana para ingresar el valor en píxeles. D. Ventana de resultado del diámetro del objeto en milímetros. 


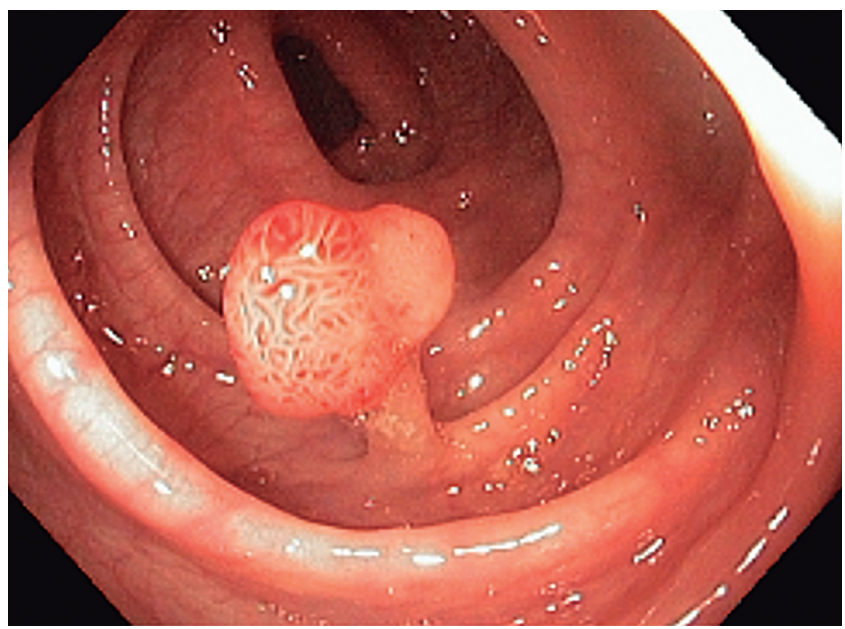

Figura 7. Pólipo del colon que será sometido al algoritmo. Fotografía (JGP) tomada con el colonoscopio, en una colonoscopia de rutina.

el tamaño de un pólipo encontrado en la colonoscopia, cuyo valor se muestra en la Figura 8, y que era de 11,09 mm.

En la Tabla 4 se muestran los resultados de las mediciones realizadas visualmente por los expertos comparadas con las medidas arrojadas por el algoritmo.

Los expertos estimaron que los pólipos de 7,8 y $9 \mathrm{~mm}$ medían 12,15 y $18 \mathrm{~mm}$, respectivamente.

\section{DISCUSIÓN}

En el presente estudio, se ha diseñado un algoritmo con las herramientas de MATLAB y con el que se ha podido deter-
Tabla 3. Objetos con diámetros conocidos (patrones de referencia) versus algoritmo

\begin{tabular}{lccc}
\hline $\begin{array}{c}\text { Objeto de } \\
\text { referencia }\end{array}$ & $\begin{array}{c}\text { Diámetro según } \\
\text { el algoritmo } \\
(\mathbf{m m})\end{array}$ & $\begin{array}{c}\text { Diámetro } \\
\text { externo, real } \\
(\mathbf{m m})\end{array}$ & $\begin{array}{c}\text { Diferencias en } \\
\text { las medidas } \\
\text { en } \mathbf{~ m m}\end{array}$ \\
\hline Anillo 2-001 & 2,86 & 2,78 & $+0,08$ \\
Anillo 2-002 & 3,65 & 3,61 & $+0,04$ \\
Anillo 2-003 & 4,66 & 4,46 & $+0,2$ \\
Lesión de la piel & 4,11 & 4 & $+0,11$ \\
Anillo 2-004 & 5,09 & 5,34 & $-0,25$ \\
Anillo 2-013 & 13,39 & 14,40 & $-1,01$ \\
Moneda (100 & 20,24 & 22 & $-1,76$ \\
pesos) & & & \\
\hline
\end{tabular}

minar, con alto grado de concordancia, el tamaño de objetos reales. Una vez comprobado el adecuado rendimiento del algoritmo, se estimaron los tamaños de pólipos del colon. Hasta donde investigamos, este es el primer estudio que utiliza MATLAB para estimar el tamaño de pólipos del colon. Con el método utilizado en esta investigación, un algoritmo con el software matemático MATLAB, la medición es prácticamente exacta. En los objetos con tamaños menores de $5 \mathrm{~mm}$, sobreestima su tamaño entre 0,11 y $0,08 \mathrm{~mm}$; para los objetos con tamaño de $5 \mathrm{~mm}$, subestima el tamaño en $0,25 \mathrm{~mm}$; y en objetos con tamaños de $22 \mathrm{~mm}$, subestima el tamaño en 1,76 mm. Globalmente, la medición con el algo-
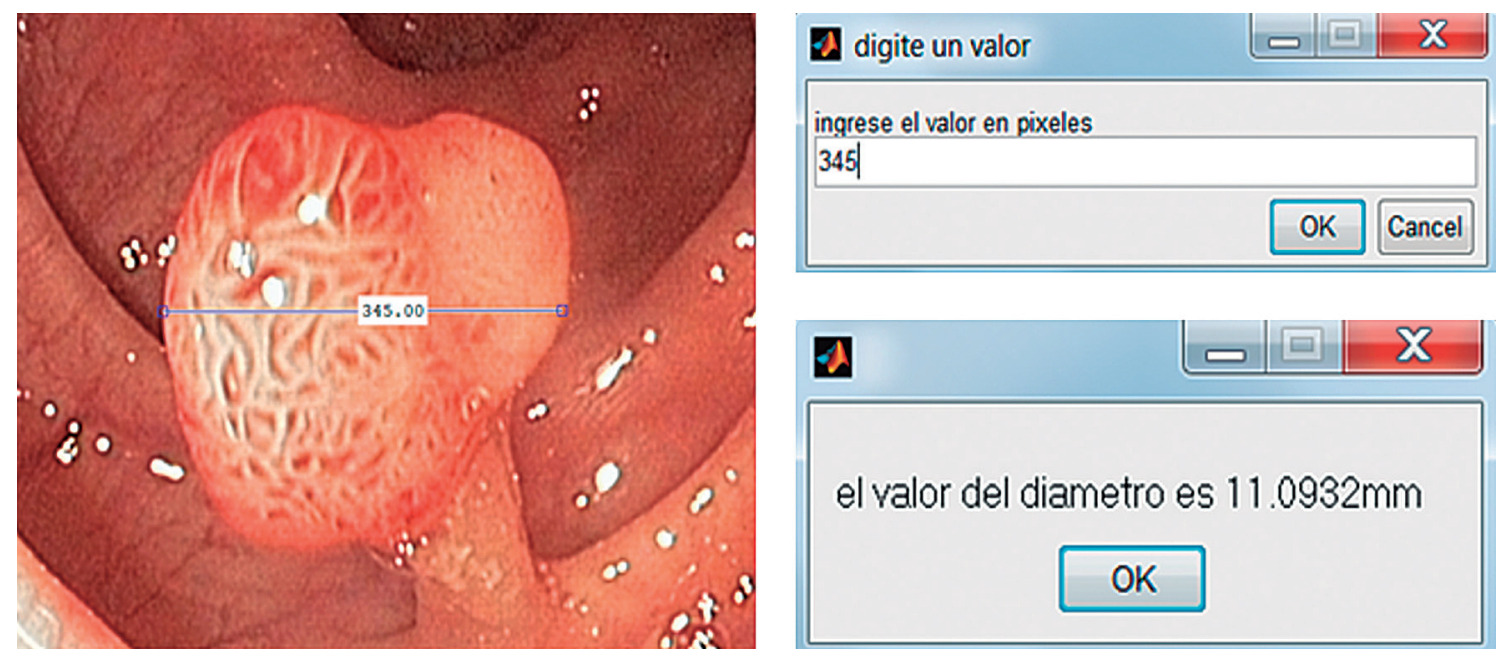

Figura 8. Pólipo sometido al algoritmo. 
Tabla 4. Tamaños de los pólipos estimados por gastroenterólogos expertos comparados con los arrojados por MATLAB

\begin{tabular}{cccccccc}
\hline $\begin{array}{c}\text { Imagen } \\
\text { JPG }\end{array}$ & $\begin{array}{c}\text { Valor según el } \\
\text { algoritmo en } \\
\text { mm }\end{array}$ & $\begin{array}{c}\text { Valor en mm } \\
\text { considerado por } \\
\text { el experto 1 }\end{array}$ & $\begin{array}{c}\text { Diferencia de las } \\
\text { medidas en mm } \\
\text { (algoritmo vs. } \\
\text { experto 1) }\end{array}$ & $\begin{array}{c}\text { Valor en mm } \\
\text { considerado por } \\
\text { el experto 2 }\end{array}$ & $\begin{array}{c}\text { Diferencia de las } \\
\text { medidas en mm } \\
\text { (algoritmo vs. } \\
\text { experto 2) }\end{array}$ & $\begin{array}{c}\text { Valor en mm } \\
\text { considerado por } \\
\text { el experto 3 }\end{array}$ & $\begin{array}{c}\text { Diferencia de las } \\
\text { medidas en mm } \\
\text { (algoritmo vs. } \\
\text { experto 3) }\end{array}$ \\
\hline Pólipo 1 & 8,0 & 12,0 & $+4,0$ & 15,0 & $+7,0$ & 12,0 & $+4,0$ \\
Pólipo 2 & 10,4 & 8,0 & $-2,4$ & 8,0 & $-2,4$ & 15,0 & $+4,6$ \\
Pólipo 3 & 7,0 & 12,0 & $+5,0$ & 12,0 & $+5,0$ & 13,0 & $+6,0$ \\
Pólipo 4 & 9,4 & 15,0 & $+5,6$ & 18,0 & $+8,6$ & 13,0 & $+3,6$ \\
Pólipo 5 & 11,2 & 15,0 & $+3,8$ & 16,0 & $+4,8$ & 15,0 & $+3,8$ \\
\hline
\end{tabular}

ritmo tiene una inexactitud del $0,2 \%$ al $8 \%$ en los objetos de $22 \mathrm{~mm}$. Esta variación es impresionantemente menor que la producida por la estimación visual de los expertos, que es del $50 \%$ hacia arriba o hacia abajo (21). Con este rendimiento, consideramos que el algoritmo podría resolver la imposibilidad de medir los pólipos del colon y que su implementación tendría utilidad en gastroenterología y endoscopia, por tratarse de un método con buen rendimiento, económico y de fácil implementación. Cuando se comparó la estimación visual de los expertos en nuestro estudio, tomando como patrón de referencia el algoritmo, la medición que estos hicieron varió entre un $50 \%$ y un $80 \%$ por encima de los pólipos de $8 \mathrm{~mm}$, con valores de $12 \mathrm{~mm}, 15 \mathrm{~mm}$ y $12 \mathrm{~mm}$, respectivamente. En los pólipos de $7 \mathrm{~mm}$, los expertos dieron valores de $12 \mathrm{~mm}, 12 \mathrm{~mm}$ y $13 \mathrm{~mm}$, respectivamente, con una variación por encima del diámetro real del $50 \%$ y del $80 \%$. En los pólipos de 11,2 $\mathrm{mm}$, la sobrestimación fue del $33 \%$ al $40 \%$. Si nos basamos en la medición de estos tres expertos, los pólipos enviados y que tenían diámetros de 7 y $8 \mathrm{~mm}$ serían programados para hacerles colonoscopia en los próximos tres años, ya que el valor de los pólipos pequeños en cuestión era superior a 10 $\mathrm{mm}$. Con ese error, de sobreestimar el verdadero tamaño, se incrementan los costos y se congestionan los servicios de endoscopias a causa de las múltiples programaciones innecesarias, generadas por solicitudes basadas en tamaños erróneamente informados, superiores a $10 \mathrm{~mm}$, con lo cual, los pacientes serían sometidos a una colonoscopia en un año $(10,11)$ y esto representaría costos injustificados por el gran número de colonoscopias indicadas a nivel nacional. Los resultados obtenidos en esta investigación permiten concluir que la exactitud lograda es superior a los diferentes métodos hasta el momento utilizados.

Es importante destacar que, aunque MATLAB dispone de la opción de procesamiento o segmentación de imágenes, esta no se utilizó debido a que el valor de los píxeles contenidos dentro del objeto; es decir, en este caso, los pólipos; es similares o igual al valor de los píxeles que están en el fondo y alrededor del objeto (mucosa del colon) y no lo puede diferenciar y "aislarlo" de los valores del entorno. En un estudio reciente que utilizó el método de procesamiento de imágenes, se logró seleccionar el pólipo del entorno, haciendo uso de un láser (20); sin embargo, esta tecnología no es de fácil aplicación por su complejidad y sería muy costosa.

Es pertinente destacar que un importante indicador de calidad de la colonoscopia es la adherencia a las recomendaciones de las guías sobre colonoscopias de seguimiento pospolipectomías (31). La falta de adherencia puede incluso minimizar el impacto de una alta tasa de detección de adenomas, considerado uno de los indicadores más fuertes de calidad de una colonoscopia (32). Los intervalos de seguimiento dependen del número de pólipos, de su histología y de su tamaño $(11,31,32)$. Las actuales guías europeas recomiendan que cuando se encuentran uno o dos pólipos con tamaños menores de $10 \mathrm{~mm}$, la próxima colonoscopia de vigilancia sería en diez años; en cambio, si hay tres pólipos pequeños o por lo menos un pólipo de $10-20 \mathrm{~mm}$, la colonoscopia debería hacerse a los tres años (11). Las guías estadounidenses tienen recomendaciones similares (21). De esta manera, la medición del tamaño de los pólipos del colon es fundamental para poder planear las colonoscopias de vigilancia, ya que este se correlaciona con el riesgo de $\operatorname{CCR}(5,11,31,32)$.

\section{CONCLUSIONES}

Con el algoritmo desarrollado con el software matemático MATLAB se ha logrado una excelente aproximación del tamaño real de los objetos con mediciones conocidas, con un margen de error menor del $10 \%$ en el peor de los casos. Extrapolado este algoritmo a los pólipos del colon, se logró su medición de manera relativamente fácil. Cuando este algoritmo se utilizó como la prueba de referencia, las medi- 
ciones de los expertos, al igual que en los estudios internacionales, sobreestimaron el tamaño de las lesiones, con lo cual, teóricamente se harían colonoscopias innecesarias.

Con base en los resultados de este estudio, se infiere que la implementación de este método podrá tener un gran impacto en la atención y en los costos de los pacientes con pólipos del colon en gastroenterología. MATLAB, un software matemático, es relativamente fácil de manejar y económico (se puede utilizar gratuitamente en el internet), que cuenta con una versión estándar que puede comprarse. Con la ayuda de un ingeniero, es fácilmente implementable en un servicio de gastroenterología, ya que puede descargarse en cualquier computador. Por tanto, se ha desarrollado un nuevo método para medir los pólipos.

La presente es una investigación original, concebida para resolver la dificultad universal de medir con exactitud los pólipos del colon. En el momento, hemos iniciado su aplicación para medir igualmente los pólipos del esófago y del estómago.

\section{Recomendaciones}

Implementar este método en las salas de endoscopia.

\section{Conflictos de interés}

Ninguno.

\section{Financiación}

Los costos de la presente investigación fueron asumidos en su totalidad por los investigadores. No obstante haber sido contratado como un profesional externo, agradecemos inmensamente al ingeniero de sistemas y computación, de la Universidad Antonio Nariño, experto en MATLAB, Anuar Steven García Gutiérrez, por su dedicación, la disposición permanente para resolver nuestras dudas y por el entusiasmo mostrado a todas nuestras inquietudes.

\section{REFERENCIAS}

1. Ferlay J, Shin HR, Bray F, Forman D, Mathers C, Parkin DM. Estimate of worldwide burden of cancer in 2008: GLOBOCAN 2008. Int J Cancer. 2010;127(12):2893-917. doi: 10.1002/ijc.25516.

2. Torre LA, Bray F, Siegel RL, Ferlay J, Lortet-Tieulent J, Jemal A. Global cancer statistics, 2012. CA Cancer J Clin. 2015;65(2):87-108. doi: 10.3322/caac.21262.

3. Piñeros $M$, Hernández G, Bray F. Increasing mortality rates of common malignancies in Colombia: an emerging problem. Cancer. 2004;101(10):2285-92.

4. Center MM, Jemal A, Ward E. International trends in colorectal cancer incidence rates. Cancer Epidemiol Biomarkers
Prev. 2009;18(6):1688-94. doi: 10.1158/1055-9965.EPI09-0090.

5. MacInnis RJ, English DR, Hopper JL, Haydon AM, Gertig DM, Giles GG. Body size and composition and colon cancer risk in men. Cancer Epidemiol Biomarkers Prev. 2004;13(4):553-9.

6. Vogelstein B, Fearon ER, Hamilton SR, Kern SE, Preisinger AC, Leppert M, et al. Genetic alterations during colorectaltumor development. N Engl J Med. 1988;319(9):525-32.

7. European Colorectal Cancer Screening Guidelines Working Group, von Karsa L, Patnick J, Segnan N, Atkin W, Halloran $S$, et al. European guidelines for quality assurance in colorectal cancer screening and diagnosis: overview and introduction to the full supplement publication. Endoscopy. 2013;45(1):51-9. doi: 10.1055/s-0032-1325997.

8. Burges NG, Bahin FF, Bohurque MJ. Colonic polypectomy (with videos). Gastrointest Endosc. 2015;81(4):814-34. doi: 10.1016/j.gie.2014.12.027.

9. Kilgore TW, Abdinoor AA, Szary NM, Schowengerdt SW, Yust JB, Choudhary A, et al. Bowel preparation with splitdose polyethylene glycol before colonoscopy: a meta-analysis of randomized controlled trials. Gastrointest Endosc. 2011;73(6):1240-5. doi: 10.1016/j.gie.2011.02.007.

10. Lieberman DA, Rex DK, Winawer SJ, Giardiello FM, Johnson DA, Levin TR. Guidelines for colonoscopy surveillance after screening and polypectomy: a consensus update by the US Multi-Society Task Force on Colorectal Cancer. Gastroenterology. 2012;143(3):844-57. doi: 10.1053/j.gastro.2012.06.001.

11. Hassan C, Quintero E, Dumonceau JM, Regula J, Brandao C, Chaussade S, et al. Post-polypectomy colonoscopy surveillance: European Society of Gastrointestinal Endoscopy (ESGE) Guideline. Endoscopy. 2013;45(10):842-51. doi: $10.1055 / \mathrm{s}-0033-1344548$.

12. Moug SJ, Vernall N, Saldanha J, McGregor JR, Balsitis M, Diament RH. Endoscopists' estimation of size should not determine surveillance of colonic polyps. Colon Dis. 2010;12(7):646-50. doi: 10.1111/j.14631318.2009.01870.x.

13. Rex DK, Rabinovitz R. Variable interpretation of polyp size by using open forceps by experienced colonoscopists. Gastrointest Endosc. 2014;79(3):402-7. doi: 10.1016/j. gie.2013.08.030.

14. Hofstad B, Vatn M, Larsen S, Osnes M. Reliability of in situ measurements of colorectal polyps. Scand J Gastroenterol. 1992;27(1):59-64.

15. Margulies C, Krevsky B, Catalano MF. How accurate are endoscopic estimates of size? Gastrointest Endosc. 1994;40(2 Pt 1):174-7.

16. Hofstad B, Vatn M, Larsen S, Huitfeldt HS, Osnes M. In situ measurement of colorectal polyps to compare video and fiberoptic endoscopes. Endoscopy. 1994;26(5):461-5.

17. Morales TG, Sampliner RE, Garewal HS, Fennerty MB, Aickin $\mathrm{M}$. The difference in colon polyp size before and after removal. Gastrointest Endosc. 1996;43(1):25-8. 
18. Rubio CA, Höög CM, Broström O, Gustavsson J, Karlsson M, Moritz P, et al. Assessing the size of polyp phantoms in tandem colonoscopies. Anticancer Res. 2009;29(5):1539-45.

19. Watanabe T, Kume K, Yoshikawa I, Harada M. Usefulness of a novel calibrated Hood to determine indications for colon polypectomy: visual estimation of polyp size is not accurate. Int J Colorectal Dis. 2015;30(7);933-8. doi: 10.1007/ s00384-015-2203-0.

20. Oka K, Seki T, Akatzu T, Wakabayashi T, Inui K, Yoshino J. Clinical study using novel endoscopic system for measuring size of gastrointestinal lesion. World J Gastroenterol. 2014;20(14):4050-8. doi: 10.3748/wjg.v20.i14.4050.

21. Brooks DD, Winawer SJ, Rex DK, Zauber AG, Kahi CJ, Smith RA, et al. Colonoscopy surveillance after polypectomy and colorectal cancer resection. Am Fam Physician. 2008;77(7):995-1002.

22. Anguiano E. ¿Qué es una imagen digital? [Internet]. [Fecha de consulta: 27 de enero de 2015]. Disponible en: http:// arantxa ii.uam.es/ eloy/html/doctorado/doct_9.pdf.

23. Diseño de materiales multimedia. Formato de imagen [Internet]. [Fecha de consulta: 27 de enero de 2015]. Disponible en: http://www.ite.educacion.es/formacion/ materiales/107/cd/imagen/imagen0105.html.

24. masadelanta.com. Definición de qué es un pixel [Internet]. [Fecha de consulta: 27 de enero de 2015]. Disponible en: http://www.masadelante .com/faqs/pixel.
25. Diseño de materiales multimedia. Web 2.0. Imagen. Conceptos básicos de imagen digital [Internet]. [Fecha de consulta: 27 de enero de 2015]. Disponible En: http:// www.ite.educacion.es/formacion/materiales/107/cd/imagen/pdf/imagen01.pdf.

26. Diccionario de la Real Academia Española. Vigésima Segunda Edición; 2001.

27. MathWorks Blogs [Internet]. [Fecha de consulta: 11 de febrero de 2015]. Disponible en: http://blogs.mathworks.com/.

28. MATLAB on StackOverflow [Internet]. [Fecha de consulta: 11 de febrero de 2015]. Disponible en: http://stackoverflow.com/questions/tagged/matlab.

29. Matlab central [Internet]. [Fecha de consulta: 11 de febrero de 2015]. Disponible en: http://www.mathworks.com/ matlabcentral/.

30. Moore H. Matlab para ingenieros. México: Editorial Pearso Educación; 2007.

31. Rex DK, Schonfield P, Cohen J, Pike IM, Adler DG, Fennerty $\mathrm{MB}$, et al. Quality indicators for colonoscopy. Gastrointest Endosc. 2015;81(1):31-53. doi: 10.1016/j.gie.2014.07.058.

32. Rees CJ, Bevan R, Zimmermann-Fraedrich K, Rutter MD, Rex D, Dekker E, et al. Expert opinions and scientific evidence for colonoscopy key performance indicators. Gut. 2016;65(12):2045-60. doi: 10.1136/gutjnl-2016-312043. 